\title{
Quantitative Evaluation of the Effect of Attenuation Correction in SPECT Images with CT-Derived Attenuation
}

\author{
Meysam Tavakoli $^{a}$ and Maryam Naji ${ }^{b}$ \\ ${ }^{a}$ Department of Physics, Indiana University-Purdue University, Indianapolis, IN 46202; \\ ${ }^{b}$ Department of Nuclear Medicine, Imam reza Hospital, Mashhad, IRAN
}

\begin{abstract}
In this study, we assessed the importance of attenuation correction by quantitative evaluation of errors associated with attenuation in myocardial SPECT in a phantom study. To do attenuation correction we use an attenuation map derived from X-ray CT data. The succession of attenuation correction highly depends on high quality of attenuation maps. CT derived attenuation map in related to non-uniform attenuation correction is used to do transmission dependent scatter correction. The OSEM algorithm with attenuation model was developed and used for attenuation correction during image reconstruction. Finally a comparison was done between reconstructed images using our OSEM code and analytical FBP method. The results of measurements show that: Our programs are capable to reconstruct SPECT images and correct the attenuation effects. Moreover to evaluate reconstructed image quality before and after attenuation correction we applied a famous approach using Image Quality Index. Attenuation correction increases the quality and quantity factors in both methods. This increasing is independent of activity in quantity factor and decrease with activity in quality factor. Both quantitative and qualitative of SPECT images were improved by attenuation correction. In both OSEM and FBP the activity ratio of heart phantom in comparison with the markers was very increased. So the attenuation correction in fat patients and low activity is recommended. Attenuation correction with CT images and OSEM reconstruction in the condition of complete registration yields superior results.
\end{abstract}

Keywords: Attenuation correction, OSEM image reconstruction, SPECT, Attenuation Map, Image Quality Index.

\section{INTRODUCTION}

In quantitative single-photon emission computed tomography (SPECT), the concentrations of absolute radionuclide is determined that allows important information to be acquired regarding in vivo function. Due to some degrading effects such as attenuated and scattered photons, this information has not been available from SPECT studies. ${ }^{1}$ Additionally, quantitative data from structures that have sizes less than around three times bigger than the total system spatial resolution can also be affected by the partial volume effect. ${ }^{2}$ In this case, computed tomography $(\mathrm{CT})$ has been used as an accurate imaging system to use for non-uniform attenuation correction. ${ }^{3-5}$ This approach relies on the use of an attenuation $\mu$ map which is produced from the CT data applying a conversion from Hounsfield units to attenuation coefficients. This method for attenuation correction has become very practical by presenting combination of SPECT/CT systems, and offers the additional information on fusing high-resolution anatomical structures together with functional images. ${ }^{6}$

In myocardial perfusion studies, the diagnostic application of using SPECT on myocardial perfusion imaging is well established to diagnosis risk-stratified patients for possibility of myocardial infarction or cardiac death, coronary artery disease, and guiding management decisions for people with known or suspected cases for coronary artery disease. ${ }^{7,8}$ However, the full clinical potential of SPECT has not been understood due to many factors that cause artifacts. ${ }^{9}$ These artifacts reduce image quality and consequently, increase the mistakes on the decisions. As we mentioned above, the important factors which decrease the quality of SPECT images are attenuation of photon, geometric detector response, and scattering. Suitable corrections for scatter and attenuation of photon are essential for quantitative studies in SPECT. Accurate attenuation correction can be performed if we access to body attenuation map. This map can be acquired using either an external source or CT.

Further author information: (Send correspondence to Meysam Tavakoli)

E-mail: mtavakol@iu.edu, mtavakol@purdue.edu,

Medical Imaging 2019: Physics of Medical Imaging, edited by Taly Gilat Schmidt, Guang-Hong Chen, Hilde Bosmans, Proc. of SPIE Vol. 10948, 109485U · (C) 2019 SPIE · CCC code: 1605-7422/19/\$18 · doi: 10.1117/12.2512120 


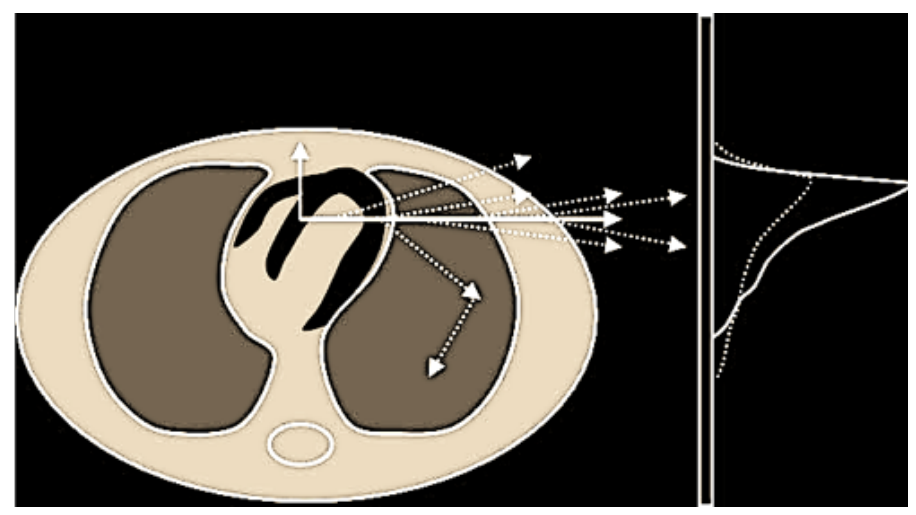

Figure 1. Photon attenuations and their effects on measured projections. Lines, photoelectric absorption. Dash lines, Compton scattering show different possible paths. For more information see ${ }^{8}$

\subsection{Physics of Attenuation}

Photon Attenuation within the body is normally accepted as the physical phenomena that affects interpretation and quantitative accuracy of myocardial perfusion SPECT images. ${ }^{9-11}$ Physical models of attenuation are characterized by a complex set of energy and tissue dependent interactions as photons traverse the body. ${ }^{12}$ The range of energies that single photon emits radioisotopes, $(70-360 \mathrm{keV})$, and physical characteristics of tissues describing the predominant effects are photoelectric absorption and Compton scattering interactions. ${ }^{12,13}$ Attenuation is an exponential procedure introduced by the linear attenuation coefficient $\mu$, expressed normally in units of $\mathrm{cm}^{-1}$, and expresses the probability per unit path length that interactions will happen. ${ }^{12}$ When attenuation coefficient, $\mu$, is determined, the value acquired depends on the portion of scatter included in the measurement. ${ }^{12}$ In CT, $\mu$ is represented in Hounsfield units relating all $\mu$ values to the value for water.15 Attenuation interactions also can be specified by the half-value layer, which presents the path length needed to reduce the intensity of beam by $50 \% \cdot{ }^{12,13}$ For the proper energy range, these values are around 4 to $6 \mathrm{~cm}$ in soft tissue and show the considerable impact of attenuation. In SPECT, because the spatial distribution of $\mu$ and its impact on the projection images is unknown, more information is needed to revise these effects. Therefore, independent measurement of the attenuation distribution is used with new reconstruction algorithms to do attenuation correction. Fig. 1 shows the effect of photoelectric absorption and Compton scattering and the variety values of $\mu$ in the thorax.

\subsection{Iterative Reconstruction}

There are several advantages when Attenuation map generated from CT images such as improved image quality and shorter acquisition time. The most important role of CT in SPECT imaging is to produce accurate nonuniform attenuation correction. Attenuation correction moved forward to clinical implementation as a result of new reconstruction methods referred to jointly as iterative algorithms. ${ }^{14-17}$ Iterative algorithms supply a broad mathematical skeleton that allows the modeling of physical processes and noise properties from the emission and detection processes. ${ }^{14}$ From reconstruction algorithms view point, in order to compensate the attenuation in filtered back projection (FBP) reconstruction, data should be corrected before or after reconstruction. The main problem in correcting data before reconstruction is that we need to determine compensation value for each pixel of the projections. There are some studies that explain approximate methods for attenuation correction before reconstruction. ${ }^{1,18-20}$ Applying of these methods is limited to uniform activity distribution inside uniform attenuating tissue. Using attenuation correction on reconstructed image corrects the constraint on activity distribution; but, it still has problem on uniform attenuation media. ${ }^{21}$ There are some methods to correct the reconstructed images for non-uniform attenuating media $;^{22-25}$ however, in the case of noise reinforcement, specifically at the center of objects, it is more affected by attenuation. On the other hand, iterative reconstruction methods are better suited for attenuation correction because of more realistic models of them to produce mathematical projections and consequently realistic reconstructed images. ${ }^{11,17,24,26-29}$ The most common iterative method in SPECT is maximum likelihood expectation maximization (MLEM). ${ }^{16,17}$ Nowadays, ordered-subsets 
expectation maximization (OSEM) which is a fast implementation of MLEM is commonly used. . $^{30-33}$

The aim of current study is to establish a comprehensive quantitative approach using CT-derived corrections for attenuation, validated in the clinical setting. Here, we introduced a method for incorporating attenuation map during OSEM. We evaluated the effect of attenuation correction of SPECT images in heart phantom with CT derived attenuation.

\section{PROPOSED METHOD}

\subsection{Phantom study}

In this study a heart phantom based on SPECT system was created. This phantom has 4 fillable cylinders. Three cylinders were filled with water, as soft tissue and inner parts of heart, and one cylinder was filled with ${ }^{99 m} T c$, as a heart wall. To determine the reference image, the phantom was filled with $0.3 \mathrm{mCi}{ }^{99 m} \mathrm{Tc}$ and for 8 minutes high count planar image was acquired with matrix size of $256 \times 256$. The SPECT imaging was in 32 projections ( 25 seconds for each) with $64 \times 64$ matrix size and activities of $0.3,0.5,1$, and $2 \mathrm{mCi}$. We used different thickness for the attenuator using 2 sizes of breast and 2 different bolus sizes, $(1.1 \mathrm{~cm}$ and $2 \mathrm{~cm})$.

\subsection{Image Reconstruction}

All of the SPECT images were reconstructed using both FBP and iterative OSEM reconstruction methods. For the FBP reconstruction we did reconstructions with all current filters.

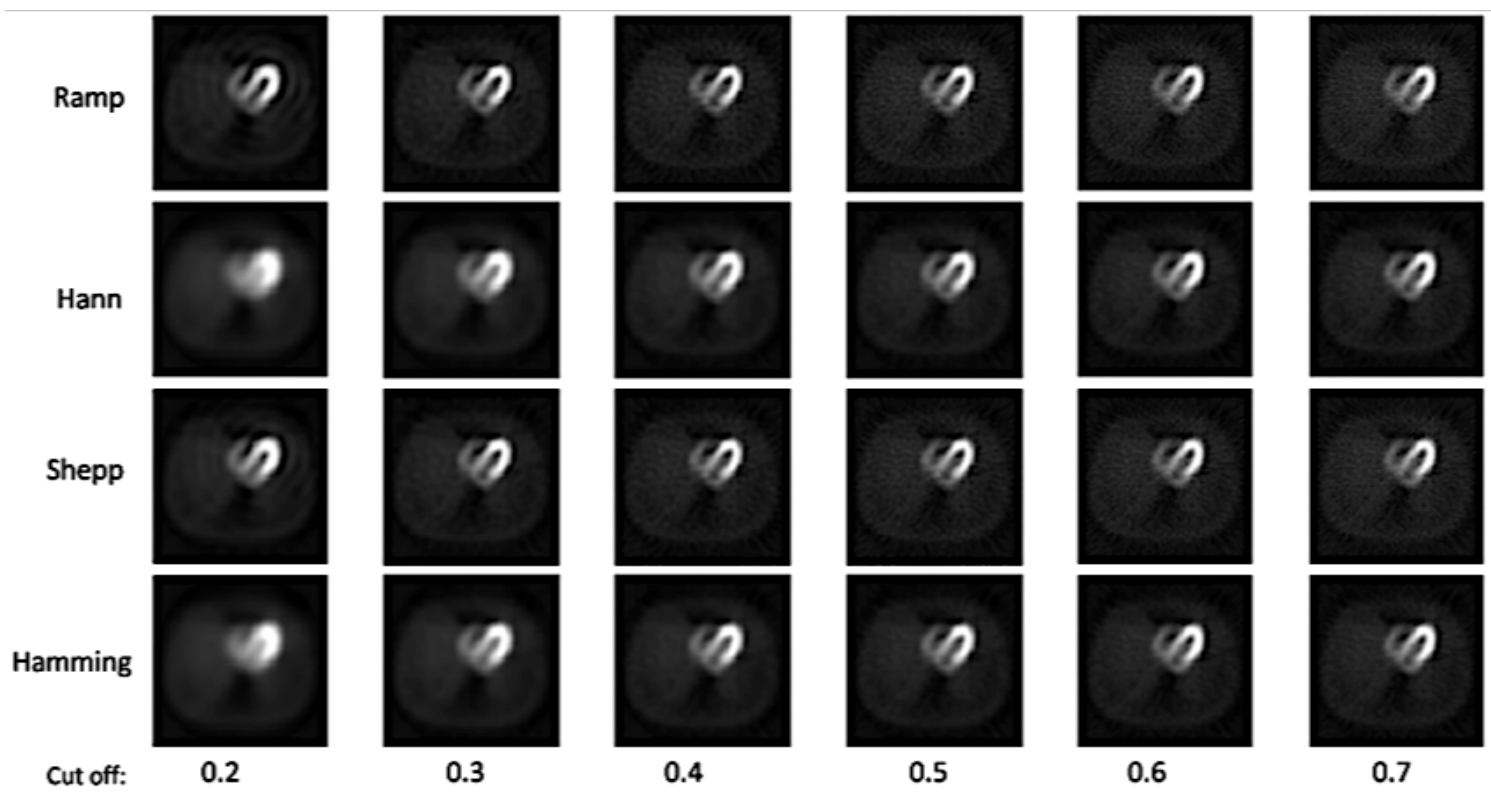

Figure 2. Reconstructed image using filtered back projection algorithm with filters implemented in MATLAB with certain cutoffs.

The Raw data were also reconstructed using the OSEM algorithm. In this part our 32 projections were classified in 4 sets of 8 projections. This means 16 angles per set for 360 degrees and 8 angles per set for 180 degrees SPECT. It is clear that the reconstructed image uniformity with OSEM changes as a function of iteration number. Moreover, for system matrix we used rectangular cross section model. ${ }^{34}$ In this model we assumed that the cross section area of pixel $j$ and detector $i$ view is proportional to the probability of detection of emitted photon from pixel $j$ in detector $i$. 


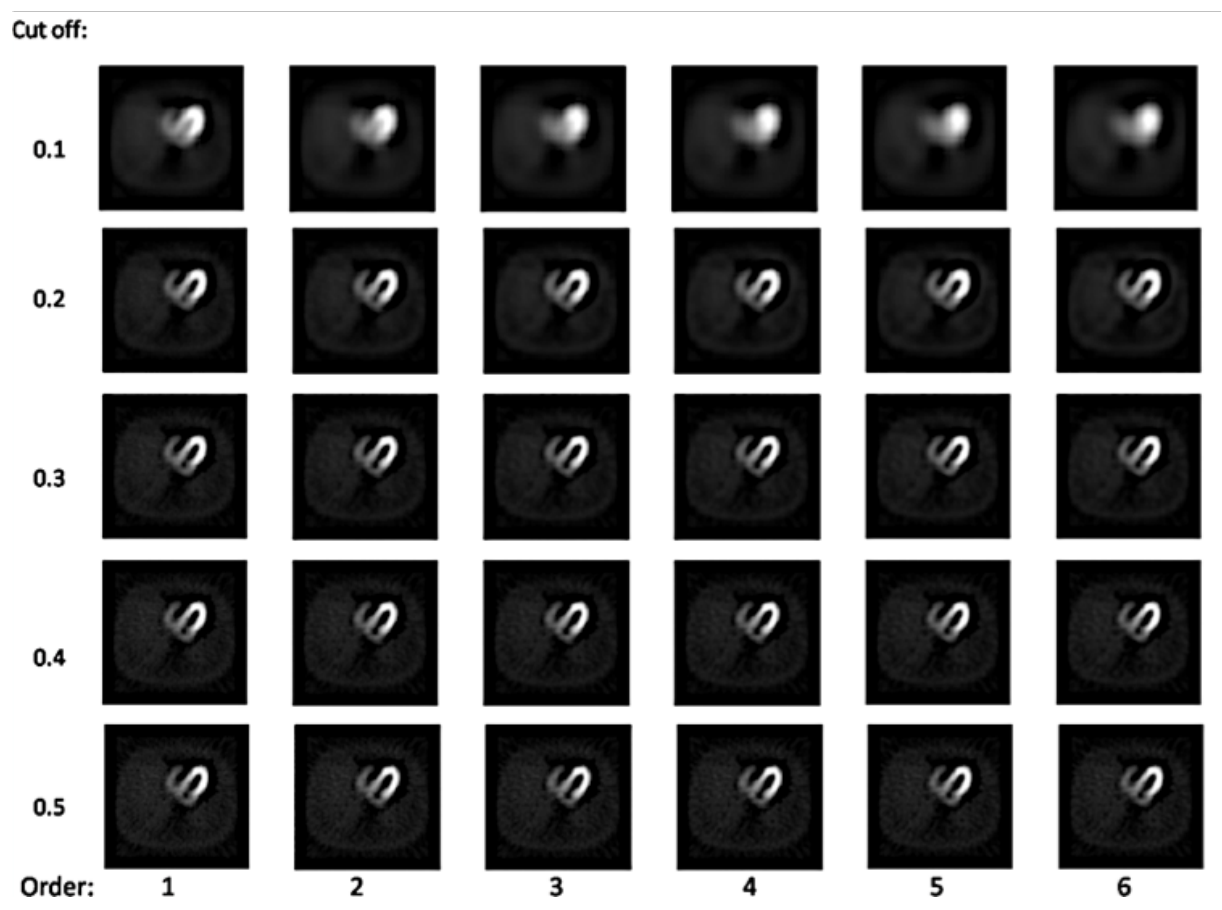

Figure 3. Reconstructed image using filtered back projection algorithm with Butterworth filter implemented in MATLAB with certain cutoffs.

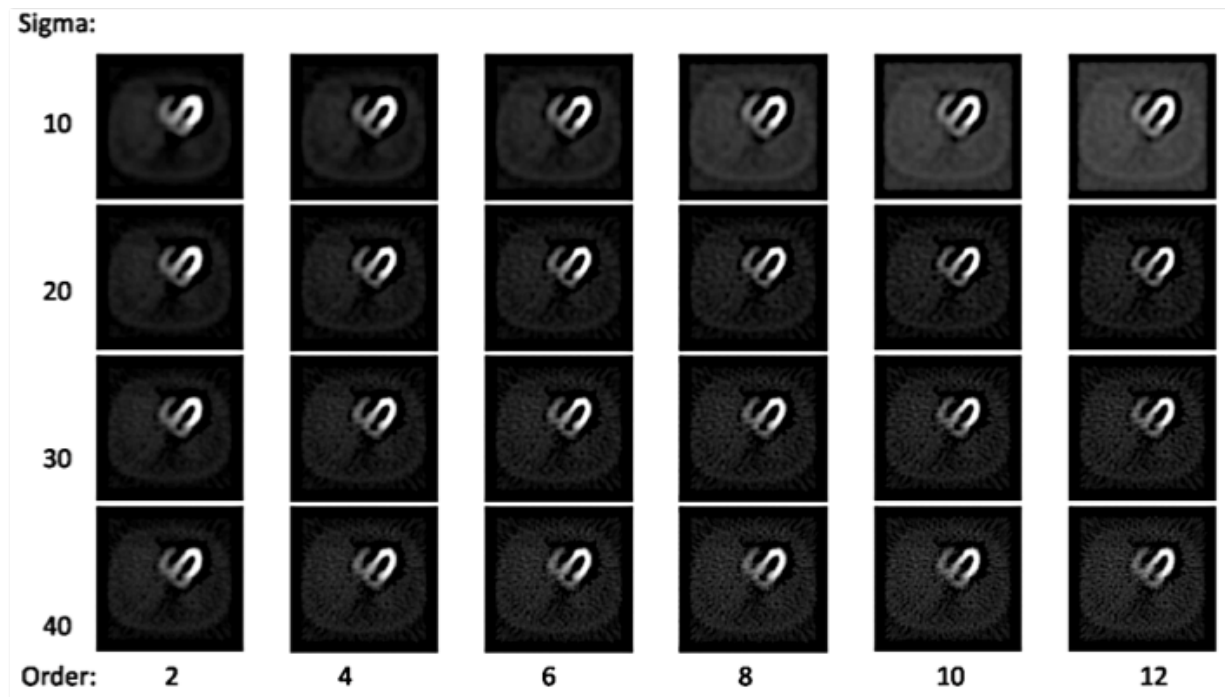

Figure 4. Reconstructed image using filtered back projection algorithm with Metz filter implemented in MATLAB with specific cutoffs.

\subsection{Determination of attenuation map}

There are number of methods for determination of attenuation map. However, use of CT is the most precise techniques to calculate attenuation map. ${ }^{19}$ In this study we used CT scanner from Siemens Company with 120 $\mathrm{keV}$ and $50 \mathrm{mAs}$ to determine attenuation map. In CT images after 2 steps the attenuation map was determined: (1) registration of CT with SPECT images using some markers, (2) converting CT numbers to linear attenuation coefficients for the emitted photons of ${ }^{99 m} \mathrm{Tc}$. For registration part at first two tubes as our markers was selected after that adjustment of resolution in SPECT and CT images. In the SPECT our acquisition matrix was $64 \times 64$ 


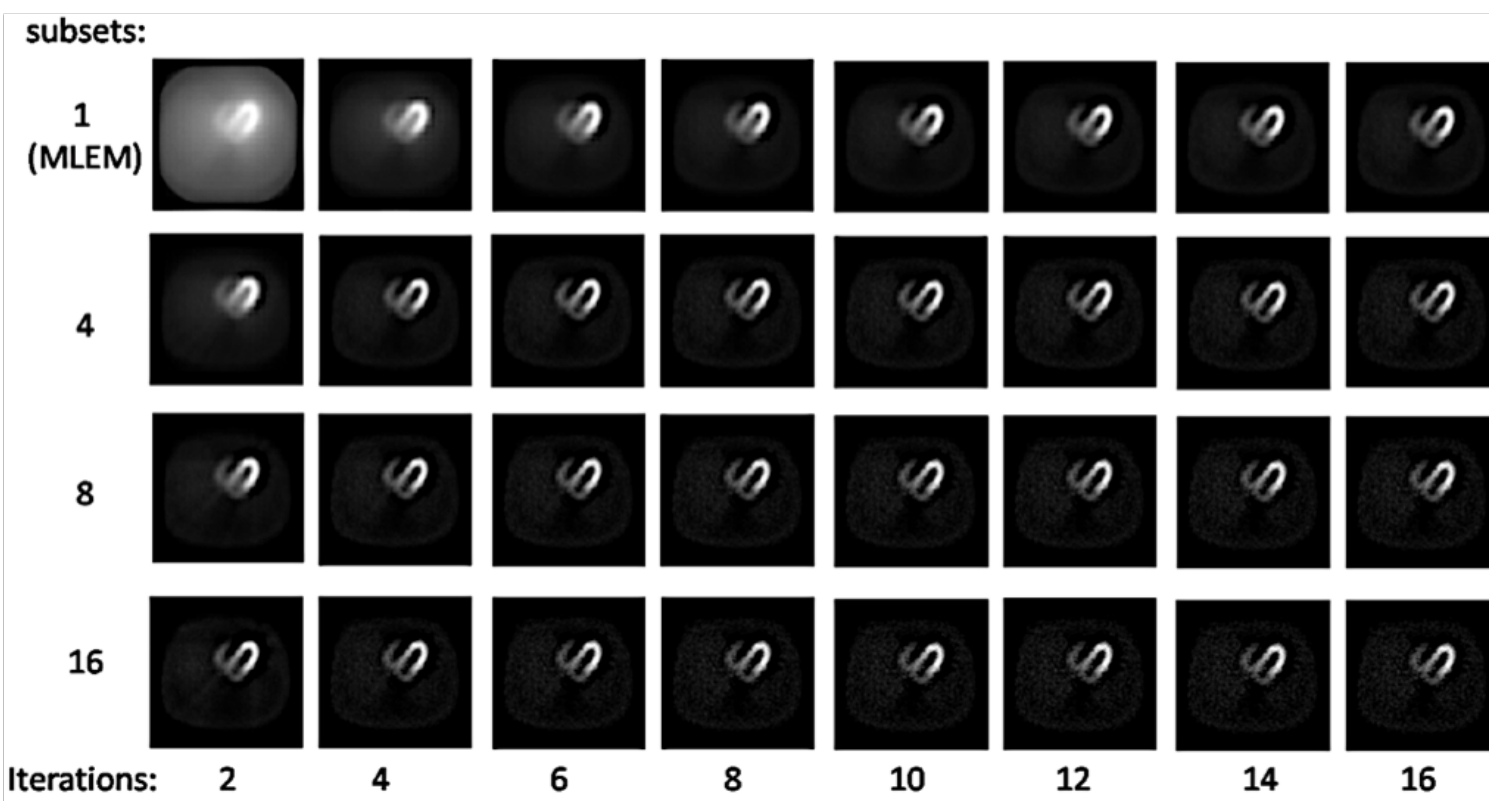

Figure 5. Reconstructed image using OSEM algorithm with different number of iterations and subsets implemented in MATLAB.

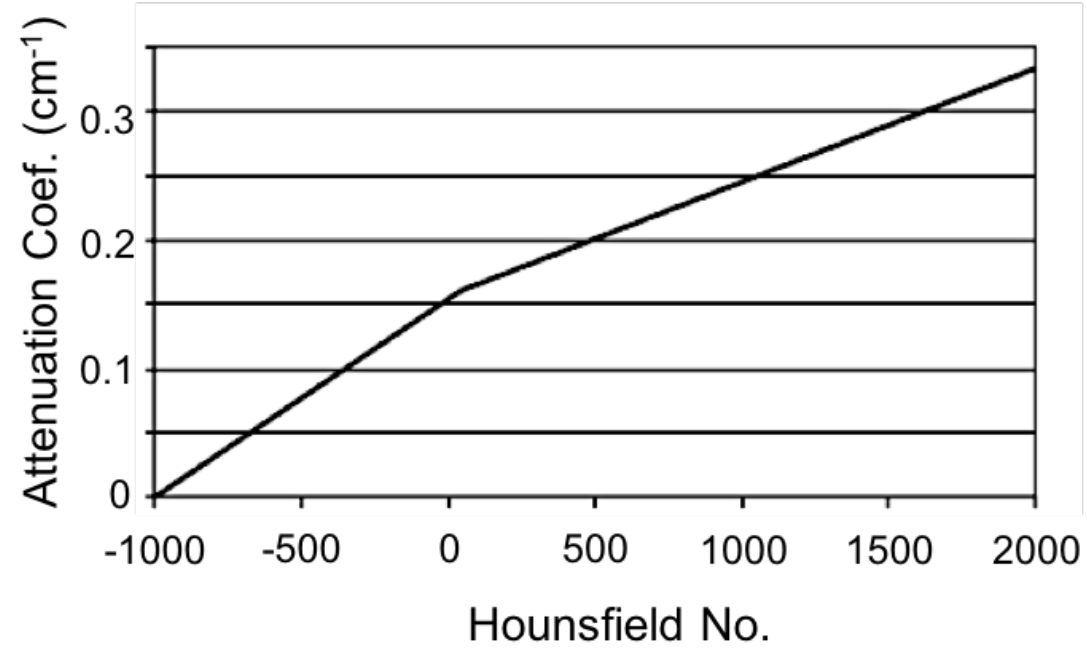

Figure 6. . Calibration curve for transferring CT numbers to attenuation coefficients of $140 \mathrm{keV}$ photons [14].

and in CT it was $256 \times 256$, so the converting factors for these two kinds of images were calculated. For this we know: Field of View $($ FOV $)=$ matrix size $\times$ pixel size.

$$
\begin{gathered}
(F O V)_{S P E C T}=64(\text { pixel }) \times 6.60(\mathrm{~mm} / \text { pixel }- \text { size })=422.40 \mathrm{~mm} \\
(F O V)_{C T}=512(\text { pixel }) \times 0.78(\mathrm{~mm} / \text { pixel }- \text { size })=399.36 \mathrm{~mm}
\end{gathered}
$$

Therefore, the transferring factor for these two imaging modalities is: $\frac{422.40}{399.36}=1.06$. In the second step we converted CT numbers to linear attenuation coefficients for the emitted photons of ${ }^{99 m} \mathrm{Tc}$ using calibration curve by Larsson. ${ }^{35}$

According to this curve, converting factor for CT numbers is in following:

$$
\mu(j)=9.05 \times 10^{-5} H U(j)+0.154(H U \geq 0)
$$



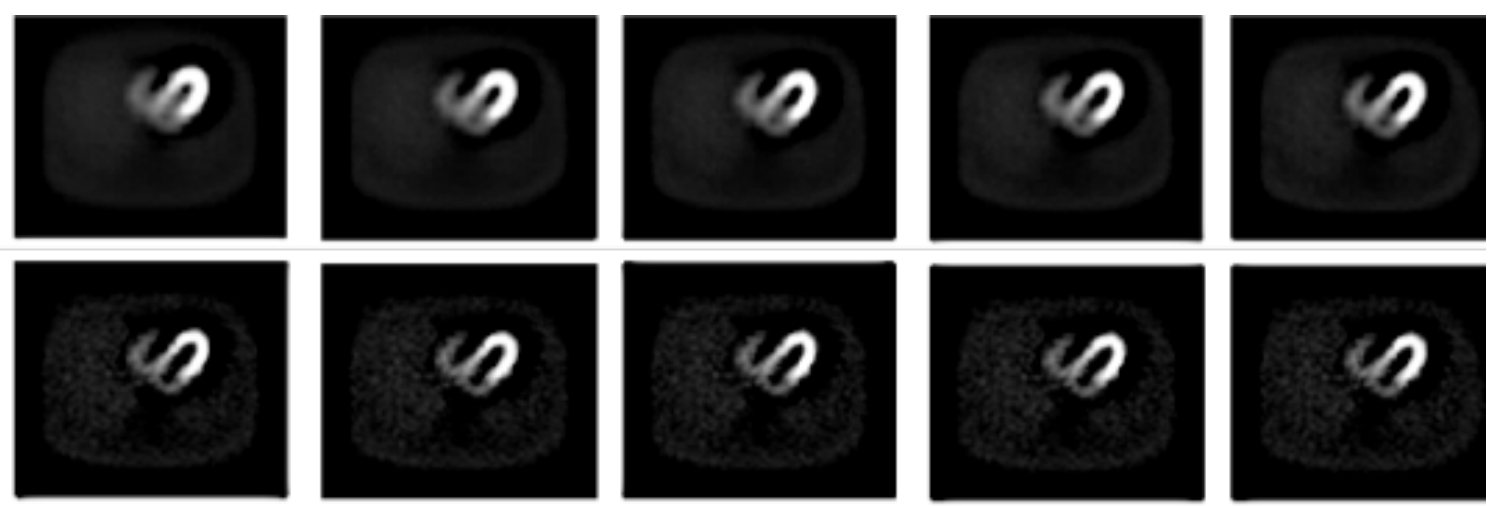

Figure 7. OSEM reconstructed image. Before attenuation correction (top) after attenuation correction (bottom).

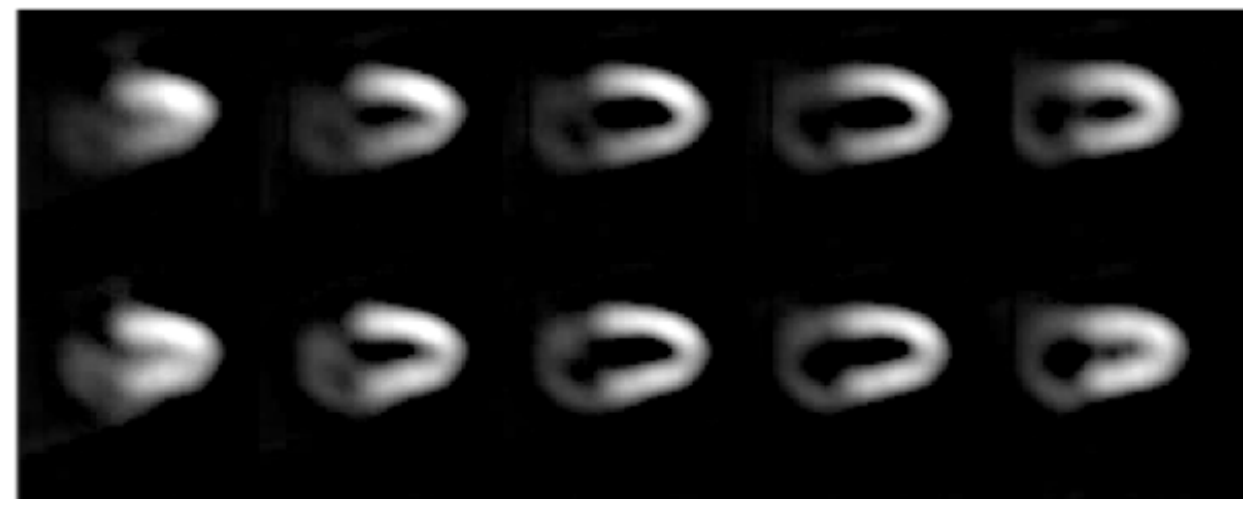

Figure 8. FBP reconstructed image. Before attenuation correction (top) after attenuation correction (bottom).

$$
\mu(j)=1.54 \times 10^{-4} H U(j)+0.154(H U<0)
$$

Where $\mathrm{HU}(j)$ is Hounsfield numbers of pixel $j$ in $120 \mathrm{keV}$ and $\mu(j)$ linear attenuation coefficient, $\mathrm{cm}^{-1}$.

\subsection{Attenuation correction algorithm}

In this section we used reconstructed images by means of system matrix. Using this attenuation map, in each angle, attenuation factor was calculated for each pixel as:

$$
\exp (\mu x(i, j))=\exp \sum_{q=1}^{k-1}-\mu(N(q) L(i, N(q))-0.5 \mu(j) L(i, j)
$$

According to this equation we calculated the attenuation of pixel $\mathrm{j}$ in bin $\mathrm{i}$ where $\mathrm{N}$ is number of pixels which were in direction of detector i view, $\mathrm{L}(i, j)$ is length of detector $i$ in pixel $j, \mathrm{k}$ is the kth pixel in direction seen by detector; therefore, the attenuation from first pixel to $(k-1)^{t h}$ was contributed in the attenuation of photon and the half of path length in pixel $j$. The attenuated system matric was determined by:

$$
a_{i j}=(\text { cross }- \text { section }- \text { area })_{i j} \times \exp \left(\mu x_{i j}\right)
$$

or The probability of detection times attenuation

\subsection{Image quality and quantity assessment}

To evaluate reconstructed image quality before and after attenuation correction we used a reference image using Image Quality Index. ${ }^{36,37}$ We determined the image quality index using:

$$
Q=\frac{4 \sigma_{x y} \bar{x} \bar{y}}{\left(\sigma_{x}^{2}+\sigma_{x}^{2}\right)\left(\bar{x}^{2}+\bar{y}^{2}\right)}
$$


Where $x$ and $y$ are mean pixel values of the test and reference images, $\sigma_{x}$ and $\sigma_{y}$ are standard deviation of test and reference images, and $\sigma_{x y}=\frac{1}{N-1} \sum_{i=1}^{N}\left(x_{i}-\bar{x}_{i}\right)\left(y_{i}-\bar{y}_{i}\right)$

This index compare test image with reference image so the more similarity the more value for this index. At last we determined quality factor by the ratio of this index for corrected and uncorrected images. For quantity assessment purpose, by using reference image we determined system response after that by help of this response, activities of different parts of the image converted to counts. We determined quantity factor by the ratio of the counts for corrected and uncorrected images.

\section{RESULTS}

Quantitative and qualitative factors are increased with attenuation correction in both FBP and OSEM reconstructed image. Quantitative factor for phantom changed between 3.3 to 5.1 for minimum and maximum attenuation respectively. The qualitative factor varied from 10 to 19.5 for both minimum and maximum attenuation.

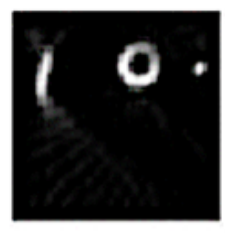

a

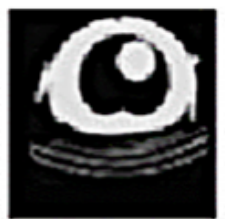

b

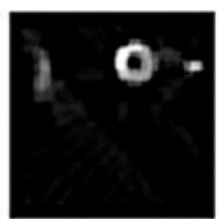

c

Figure 9. FBP reconstructed image. Before attenuation correction (a) attenuation map (b) after attenuation correction (c).

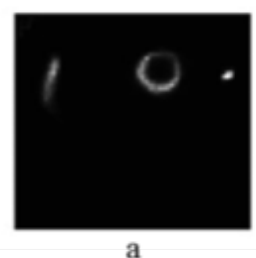

a

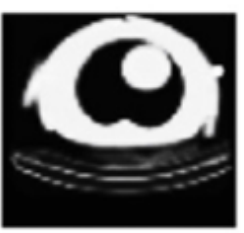

b

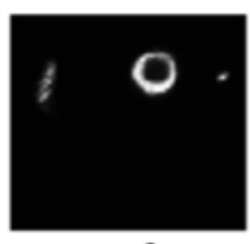

c

Figure 10. OSEM reconstructed image. Before attenuation correction (a) attenuation map (b) after attenuation correction (c).

\section{DISCUSSION AND CONCLUSION}

Overal, using Computer-Aided Diagnosis system in all medical imaging aspects would save the workload and time of well-paid clinicians and helps them in their final decisions. Also it allows hospitals to use their resources in other important tasks. ${ }^{38-40}$ In general, spatially registered SPECT images combine with CT information can be applied to correlate physiological data from the emission image with anatomical data from the transmission image. ${ }^{41}$ From quantitative studies view point, ${ }^{40} \mathrm{CT}$ images create an attenuation map to correct attenuation errors in the SPECT images. ${ }^{3,42,43}$ Moreover, if the CT images have enough resolution and contrast, they provide spatial information to correct and revise the SPECT image from partial volume errors. ${ }^{4,43}$ Computer simulations and phantom study have found that CT-derived attenuation maps with as great as $6 \%$ errors result in SPECT images in which the myocardial radionuclide concentration is accurate to within $1 \%$ and only insignificant artifacts are seen in bone regions. Some studies ${ }^{44,45}$ have demonstrate that the signal-to-noise ratio in CT images is above that needed for attenuation correction of SPECT. ${ }^{4}$ The CT-derived attenuation map for the torso phantom is more than these empirically-based needs for attenuation correction in both accuracy and precision. The important 
limitation of SPECT/CT systems in providing quantitative data about the activity distribution is related to the severe degradation of the acquired information by different physics effects. ${ }^{25,46,47}$ One of these image-degrading issues is attenuation of photon as it passes through different layers of tissue, and loss of resolution because of collimator blurring. The relative impact of this problem in the SPECT quantitative accuracy was investigated in some studies. ${ }^{47,48}$ Combination of an accurate explanation of physics phenomena into reconstruction methods provides an useful way to improve quantitative properties of the recovered image. ${ }^{49,50}$

In this study, both quantitatively and qualitatively of SPECT images were improved by attenuation correction. Without attenuation correction, the SPECT images of the phantom reconstnicted is not accurate both in absolute quantitation and in relative intensities within the image. In both OSEM and FBP the activity ratio of heart phantom in comparison with the markers is very increased. For example, this ratio for activity of $0.3 \mathrm{mCi}$, for phantom itself changes from 1.20 to 3.76 and for phantom plus breast and bolus changes from 1.36 to 5.1. According to our results for effects of low and high activities, qualitative values are increases when we increase the size of attenuators and consequently that means the correction is better. The pixels near to the attenuator have higher attenuation because of the principle that when distance is increased in each projection the probability of photon passing through tissue is decreased. It seems qualitative values are decreased when activity increased and quantitative values are independent of activity. According to the results, attenuation correction is more effective in low activity images (or low count) or with thick attenuator. Moreover, we recommend accurate registration of CT and SPECT images because of important role of attenuator image.

\section{ACKNOWLEDGMENTS}

Meysam Tavakoli would like to thank Dr. Faraz Kalantari for his valuable discussion for final preparation of the manuscript.

\section{REFERENCES}

1. K. Willowson, D. L. Bailey, and C. Baldock, "Quantitative SPECT reconstruction using CT-derived corrections," Physics in Medicine ES Biology 53(12), p. 3099, 2008.

2. E. J. Hoffman, S.-C. Huang, and M. E. Phelps, "Quantitation in positron emission computed tomography: 1. effect of object size.," Journal of computer assisted tomography 3(3), pp. 299-308, 1979.

3. K. LaCroix, B. Tsui, B. Hasegawa, and J. Brown, "Investigation of the use of X-ray CT images for attenuation compensation in SPECT," IEEE Transactions on Nuclear Science 41(6), pp. 2793-2799, 1994.

4. S. Blankespoor, X. Xu, K. Kaiki, J. Brown, H. Tang, C. Cann, and B. Hasegawa, "Attenuation correction of SPECT using X-ray CT on an emission-transmission CT system: myocardial perfusion assessment," IEEE Transactions on Nuclear Science 43(4), pp. 2263-2274, 1996.

5. T. Kashiwagi, K. Yutani, M. Fukuchi, H. Naruse, T. Iwasaki, K. Yokozuka, S. Inoue, and S. Kondo, "Correction of nonuniform attenuation and image fusion in SPECT imaging by means of separate x-ray ct," Annals of nuclear medicine 16(4), pp. 255-261, 2002.

6. M. Bocher, A. Balan, Y. Krausz, Y. Shrem, A. Lonn, M. Wilk, and R. Chisin, "Gamma camera-mounted anatomical X-ray tomography: technology, system characteristics and first images," European journal of nuclear medicine 27(6), pp. 619-627, 2000.

7. S. Underwood, C. Anagnostopoulos, M. Cerqueira, P. Ell, E. Flint, M. Harbinson, A. Kelion, A. AlMohammad, E. Prvulovich, L. Shaw, et al., "Myocardial perfusion scintigraphy: the evidence," European journal of nuclear medicine and molecular imaging 31(2), pp. 261-291, 2004.

8. T. M. Bateman and S. J. Cullom, "Attenuation correction single-photon emission computed tomography myocardial perfusion imaging," in Seminars in nuclear medicine, 35(1), pp. 37-51, Elsevier, 2005.

9. E. DePuey 3rd, "How to detect and avoid myocardial perfusion SPECT artifacts.," Journal of nuclear medicine: official publication, Society of Nuclear Medicine 35(4), p. 699, 1994.

10. M. A. King, B. M. Tsui, and T.-S. Pan, "Attenuation compensation for cardiac single-photon emission computed tomographic imaging: Part 1. impact of attenuation and methods of estimating attenuation maps," Journal of Nuclear Cardiology 2(6), pp. 513-524, 1995. 
11. M. A. King, B. M. Tsui, T.-S. Pan, S. J. Glick, and E. J. Soares, "Attenuation compensation for cardiac single-photon emission computed tomographic imaging: Part 2. attenuation compensation algorithms," Journal of nuclear cardiology 3(1), pp. 55-64, 1996.

12. J. A. Sorenson and M. E. Phelps, Physics in nuclear medicine, Grune \& Stratton New York, 1987.

13. J. T. Bushberg and J. M. Boone, The essential physics of medical imaging, Lippincott Williams \& Wilkins, 2011.

14. B. M. Tsui, G. T. Gullberg, E. R. Edgerton, J. G. Ballard, J. R. Perry, W. H. McCartney, and J. Berg, "Correction of nonuniform attenuation in cardiac SPECT imaging," Journal of Nuclear Medicine 30(4), pp. 497-507, 1989.

15. K. Lange, M. Bahn, and R. Little, "A theoretical study of some maximum likelihood algorithms for emission and transmission tomography," IEEE Transactions on Medical Imaging 6(2), pp. 106-114, 1987.

16. K. Lange and R. Carson, "Em reconstruction algorithms for emission and transmission tomography," $J$ Comput Assist Tomogr 8(2), pp. 306-16, 1984.

17. L. A. Shepp and Y. Vardi, "Maximum likelihood reconstruction for emission tomography," IEEE transactions on medical imaging $\mathbf{1}(2)$, pp. 113-122, 1982.

18. R. J. Jaszczak, P. H. Murphy, D. Huard, and J. A. Burdine, "Radionuclide emission computed tomography of the head with 99mcc and a scintillation camera.," Journal of nuclear medicine: official publication, Society of Nuclear Medicine 18(4), pp. 373-380, 1977.

19. H. Zaidi and B. Hasegawa, "Determination of the attenuation map in emission tomography," Journal of Nuclear Medicine 44(2), pp. 291-315, 2003.

20. Y. Masood, Y.-H. Liu, G. DePuey, R. Taillefer, L. I. Araujo, S. Allen, D. Delbeke, F. Anstett, A. Peretz, M.-J. Zito, et al., "Clinical validation of SPECT attenuation correction using x-ray computed tomographyderived attenuation maps: multicenter clinical trial with angiographic correlation," Journal of nuclear cardiology 12(6), pp. 676-686, 2005.

21. L.-T. Chang, "A method for attenuation correction in radionuclide computed tomography," IEEE Transactions on Nuclear Science 25(1), pp. 638-643, 1978.

22. M. Ljungberg and S.-E. Strand, "Attenuation correction in SPECT based on transmission studies and monte carlo simulations of build-up functions," Journal of Nuclear Medicine 31(4), pp. 493-500, 1990.

23. M. Ljungberg and S.-E. Strand, "Attenuation and scatter correction in SPECT for sources in a nonhomogeneous object: a monte carlo study," Journal of Nuclear Medicine 32(6), p. 1278, 1991.

24. H. Zaidi, "Relevance of accurate monte carlo modeling in nuclear medical imaging," Medical physics 26(4), pp. 574-608, 1999.

25. M. A. King, S. J. Glick, P. H. Pretorius, R. G. Wells, H. C. Gifford, M. V. Narayanan, and T. Farncombe, "Attenuation, scatter, and spatial resolution compensation in SPECT," Emission tomography. Academic Press, San Diego, 2004.

26. F. J. Beekman, J. M. den Harder, M. A. Viergever, and P. P. van Rijk, "SPECT scatter modelling in non-uniform attenuating objects," Physics in medicine $E$ biology 42(6), p. 1133, 1997.

27. J. Nuyts, P. Dupont, S. Stroobants, R. Benninck, L. Mortelmans, and P. Suetens, "Simultaneous maximum a posteriori reconstruction of attenuation and activity distributions from emission sinograms," IEEE transactions on medical imaging 18(5), pp. 393-403, 1999.

28. Y. Seo, K. H. Wong, M. Sun, B. L. Franc, R. A. Hawkins, and B. H. Hasegawa, "Correction of photon attenuation and collimator response for a body-contouring SPECT/CT imaging system," Journal of Nuclear Medicine 46(5), pp. 868-877, 2005.

29. M. Tavakoli, M. Naji, A. Abdollahi, and F. Kalantari, "Attenuation correction in SPECT images using attenuation map estimation with its emission data," in Medical Imaging 2017: Physics of Medical Imaging, 10132, p. 101324Z, International Society for Optics and Photonics, 2017.

30. H. M. Hudson and R. S. Larkin, "Accelerated image reconstruction using ordered subsets of projection data," IEEE transactions on medical imaging 13(4), pp. 601-609, 1994.

31. C. Vanhove, M. Defrise, P. R. Franken, H. Everaert, F. Deconinck, and A. Bossuyt, "Interest of the ordered subsets expectation maximization (os-em) algorithm in pinhole single-photon emission tomography reconstruction: a phantom study," European journal of nuclear medicine 27(2), pp. 140-146, 2000. 
32. J. Zeintl, A. H. Vija, A. Yahil, J. Hornegger, and T. Kuwert, "Quantitative accuracy of clinical 99mtc SPECT/CT using ordered-subset expectation maximization with 3-dimensional resolution recovery, attenuation, and scatter correction," Journal of Nuclear Medicine 51(6), p. 921, 2010.

33. R. G. Wells, M. A. King, P. H. Simkin, P. F. Judy, A. B. Brill, H. C. Gifford, R. Licho, P. H. Pretorius, P. B. Schneider, and D. W. Seldin, "Comparing filtered backprojection and ordered-subsets expectation maximization for small-lesion detection and localization in^ $6^{\wedge} 7 \mathrm{ga}$ SPECT," Journal of nuclear medicine 41(8), pp. 1391-1399, 2000.

34. J. A. Fessler and I. Tutorial, "Iterative methods for image reconstruction," in IEEE International Symposium on Biomedical Imaging Tutorial, Arlington Virginia, 2006.

35. A. Larsson, Corrections for improved quantitative accuracy in SPECT and planar scintigraphic imaging. $\mathrm{PhD}$ thesis, Umeå universitet, 2005.

36. Z. Wang, A. C. Bovik, H. R. Sheikh, and E. P. Simoncelli, "Image quality assessment: from error visibility to structural similarity," IEEE transactions on image processing 13(4), pp. 600-612, 2004.

37. Z. Wang and A. C. Bovik, "A universal image quality index," IEEE signal processing letters 9(3), pp. 81-84, 2002.

38. M. Tavakoli, M. Nazar, and A. Mehdizadeh, "Effect of two different preprocessing steps in detection of optic nerve head in fundus images," in Medical Imaging 2017: Computer-Aided Diagnosis, 10134, p. 101343A, International Society for Optics and Photonics, 2017.

39. M. Tavakoli, R. P. Shahri, H. Pourreza, A. Mehdizadeh, T. Banaee, and M. H. B. Toosi, "A complementary method for automated detection of microaneurysms in fluorescein angiography fundus images to assess diabetic retinopathy," Pattern Recognition 46(10), pp. 2740-2753, 2013.

40. R. Pourreza-Shahri, M. Tavakoli, and N. Kehtarnavaz, "Computationally efficient optic nerve head detection in retinal fundus images," Biomedical Signal Processing and Control 11, pp. 63-73, 2014.

41. E. L. Kramer, M. E. Noz, J. J. Sanger, A. J. Megibow, and G. Maguire, "CT-spect fusion to correlate radiolabeled monoclonal antibody uptake with abdominal CT findings.," Radiology 172(3), pp. 861-865, 1989.

42. J. Fleming, "A technique for using CT images in attenuation correction and quantification in SPECT.," Nuclear medicine communications 10(2), pp. 83-97, 1989.

43. K. F. Koral, K. R. Zasadny, M. L. Kessler, J.-Q. Luo, S. F. Buchbinder, M. S. Kaminski, I. Francis, and R. L. Wahl, "CT-SPECT fusion plus conjugate views for determining dosimetry in iodine-131-monoclonal antibody therapy of lymphoma patients," Journal of Nuclear Medicine 35(10), p. 1714, 1994.

44. S. C. Liew, B. Hasegawa, J. Brown, and T. Lang, "Noise propagation in SPECT images reconstructed using an iterative maximum-likelihood algorithm," Physics in Medicine E Biology 38(12), p. 1713, 1993.

45. C.-H. Tung and G. T. Gullberg, "A simulation of emission and transmission noise propagation in cardiac SPECT imaging with nonuniform attenuation correction," Medical physics 21(10), pp. 1565-1576, 1994.

46. B. M. Tsui, E. C. Frey, K. J. LaCroix, D. S. Lalush, W. H. McCartney, M. A. King, and G. T. Gullberg, "Quantitative myocardial perfusion SPECT," Journal of Nuclear Cardiology 5(5), pp. 507-522, 1998.

47. G. El Fakhri, I. Buvat, H. Benali, A. Todd-Pokropek, and R. Di Paola, "Relative impact of scatter, collimator response, attenuation, and finite spatial resolution corrections in cardiac SPECT," Journal of Nuclear Medicine 41(8), pp. 1400-1408, 2000.

48. B. He, Y. Du, X. Song, W. P. Segars, and E. C. Frey, "A monte carlo and physical phantom evaluation of quantitative in-111 SPECT," Physics in Medicine 83 Biology 50(17), p. 4169, 2005.

49. S. Shcherbinin, A. Celler, T. Belhocine, R. Vanderwerf, and A. Driedger, "Accuracy of quantitative reconstructions in SPECT/CT imaging," Physics in Medicine 83 Biology 53(17), p. 4595, 2008.

50. M. Tavakoli, A. Mehdizadeh, R. Pourreza, H. R. Pourreza, T. Banaee, and M. B. Toosi, "Radon transform technique for linear structures detection: application to vessel detection in fluorescein angiography fundus images," in Nuclear Science Symposium and Medical Imaging Conference (NSS/MIC), 2011 IEEE, pp. 30513056, IEEE, 2011. 